\title{
Engineering Translational Resource Allocation \\ Controllers: Mechanistic Models, Design Guidelines, and Potential Biological Implementations
}

\author{
Alexander P.S. Darlington, ${ }^{\dagger}$ Juhyun Kim, ${ }^{\ddagger}$ José I. Jiménez, ${ }^{\ddagger}$ and Declan G. \\ Bates*, \\ $\dagger$ Warwick Integrative Synthetic Biology Centre, School of Engineering, University of \\ Warwick, Coventry, UK \\ $\ddagger$ Faculty of Health and Medical Sciences, University of Surrey, Guildford, UK \\ E-mail: d.bates@warwick.ac.uk
}

\begin{abstract}
The use of orthogonal ribosomes in combination with dynamic resource allocation controllers is a promising approach for relieving the negative effects of cellular resource limitations on the modularity of synthetic gene circuits. Here, we develop a detailed mechanistic model of gene expression and resource allocation, which when simplified to a tractable level of complexity, allows the rational design of translational resource allocation controllers. Analysis of this model reveals a fundamental design trade-off; that reducing coupling acts to decrease gene expression. Through a sensitivity analysis of the experimentally tuneable controller parameters, we identify how each controller design parameter affects the overall closed-loop behaviour of the system, leading to a detailed set of design guidelines for optimally managing this trade-off. Based on our
\end{abstract}


designs, we evaluated a number of alternative potential experimental implementations of the proposed system using commonly available biological components. Finally, we show that the controller is capable of dynamically allocating ribosomes as needed to restore modularity in a number of more complex synthetic circuits, such as the repressilator, and activation cascades composed of multiple interacting modules.

\section{Keywords}

resource competition, orthogonal ribosomes, feedback control, modularity

Ensuring circuit modularity, i.e the independent and predictable functioning of different circuit processes, remains a key goal in synthetic biology. If modules are independent then they can be recombined to produce novel functions which can be predicted from previous characterisation. This approach is commonly used in electronics and computer science where complex functions are broken down into independent modules, which can be assembled to form new systems.

However, in synthetic circuits, there is often a failure of modularity, with gene circuits based on purportedly well characterised components needing iterative rounds of redesign and re-experimentation to obtain functional implementations. Modularity fails for a variety of

reasons: (i) unexpected cross talk between modules due to component re-use, ${ }^{1}$ (ii) subtle changes in gene regulation due to unforeseen effects of combining DNA sequences, ${ }^{2}$ (iii) retroactivity effects where the titration of a transcription factor to a downstream module affects the behaviour of the upstream module, ${ }^{3}$ and, (iv) the use of a common limited pool of resources for gene expression. ${ }^{4}$ Careful selection of components can ameliorate the effects of (i) by ensuring modules do not have off target effects. ${ }^{5}$ The introduction of 'insulator elements' such as ribozymes can reduce the effects of $(\mathrm{ii})^{6}$ and the development of buffer 
circuits allows loading fracture due to retroactivity (iii) to be reduced. ${ }^{7}$ The question of how to optimally manage the effects of cellular resource limitations on circuit modularity, however, remains an open problem.

During exponential growth, the concentration of RNA polymerases (RNAP) and ribosomes in the cell remains constant. This results in a fixed pool of gene expression resources. Whilst both resources are finite, numerous experimental studies have shown it is the number of free ribosomes in system which is the main limitation on gene expression. ${ }^{8,9,10,11,12}$ The sharing of this fixed resource across genes leads to a phenomenon known as gene-coupling. This results in the emergence of non-regulatory interactions between co-expressed genes, ${ }^{4}$ since each synthetic circuit module will utilise as many ribosomes as possible at any one moment, as determined by parameters such as mRNA levels or RBS strength.

To illustrate the problem mathematically, consider the number of free ribosomes as a function of the ribosome supply and demand as

$$
R\left(\phi_{1}\right)=\frac{R_{\text {total }}}{1+\phi_{1}}
$$

where $R_{\text {Total }}$ is the total number of ribosomes available and $\phi$ is the demand. ${ }^{4,10}$ Upon the addition of another demand $\phi_{2}$, the free ribosome number becomes:

$$
R\left(\phi_{1}, \phi_{2}\right)=\frac{R_{\text {total }}}{1+\phi_{1}+\phi_{2}}
$$

As $\phi>0$ in all cases, by definition $R\left(\phi_{1}, \phi_{2}\right)<R\left(\phi_{1}\right)$. This decrease in free ribosome number reduces the rate of downstream processes, such as mRNA-ribosome binding, as a consequence of the law of mass action. This leads to a decrease in other modules as a new module is induced (this is often termed coupling ${ }^{13,14}$ ): activation of one circuit module effectively inhibits other modules. In this case the supply of ribosomes (the numerator) is determined by the cell and supply is constant regardless of the demand (the denominator) i.e. there is no control of $R_{\text {Total }}$. 
To mitigate this decrease in free ribosomes upon the addition of new genes, consider a system where the supply of total ribosomes can be matched to the circuit's demand for ribosomes. Let this malleable ribosome pool be $R^{\prime}$ :

$$
R\left(\phi_{1}\right)=\frac{R_{\phi_{1}}^{\prime}}{1+\phi_{1}}
$$

Upon the addition of another gene the demand (the denominator) increases as before:

$$
R\left(\phi_{1}, \phi_{2}\right)=\frac{R_{\phi_{1}, \phi_{2}}^{\prime}}{1+\phi_{1}+\phi_{2}}
$$

However, in this ideal system we can now increase the supply of $R^{\prime}$ (i.e. increase the numerator) to make $R\left(\phi_{1}\right)=R\left(\phi_{1}, \phi_{2}\right)$. This maintains the free ribosome pool available to the circuit and so removes ribosome-mediated gene coupling.

In a previous work, we have experimentally realised a prototype of a feedback controller that can dynamically allocate more ribosomes to the circuit when required ${ }^{14}$. Increasing total ribosome number is not biologically feasible, so our controller acts to dynamically allocate the translational capacity between host and circuit genes in response to circuit demand, thus relieving the effects of resource limitations on the circuit ${ }^{14}$. This is achieved by regulating the production of a pool of quasi-orthogonal ribosomes. These specialised circuit-specific ribosomes can be created by expressing an orthogonal $16 \mathrm{~S}$ rRNA and replacement of the natural ribosome binding site in circuit genes, or other genes of interest, with complimentary synthetic ribosome binding sites. The o-16S rRNA replaces the endogenous host $16 \mathrm{~S}$ rRNA in a fraction of the host ribosomes creating a separate translational resource which is targeted to circuit genes by binding the complimentary synthetic RBS sequence. By placing the production of the o- $16 \mathrm{~S}$ rRNA under the control of a constitutively expressed repressive transcription factor which itself uses the orthogonal ribosome pool for its own translation, we created a feedback controller which produces o-16S rRNA, and hence orthogonal ribosomes, in response to circuit demand. As circuit genes are induced, they sequester o-ribosomes for 
their own expression resulting in a fall in the expression of the regulator. This relieves the repression of the o-16S rRNA, resulting in increased o-16S rRNA production and increased o-ribosome co-option. Thus the controller implements a negative feedback loop. See Figure 1a for a schematic of the biological implementation of this feedback loop. Of course, the controller cannot mitigate intrinsic limitations arising from the fact that the total number of ribosomes in the cell is finite. Rather, the controller acts to dynamically manage the allocation of translational activity between host and circuit genes in the most efficient way, by increasing circuit capacity as circuit demand requires it.

In this paper, we use the biological insights gained from the prototype to produce simple models of the controller architecture which can be used to inform detailed design of the systems dynamics using control theory metrics. Using such models, we demonstrate how improved resource allocation controllers can be rationally designed to decouple the expression of different genes, and develop design rules for how the tuning of different controller design parameters can act to separately specify either the dynamic response time or overall protein output (i.e. gain) of the circuit. Based on these design rules, we identify and evaluate a number of alternative potential experimental implementations of the proposed translational controllers. Finally, we demonstrate the potential of resource allocation controllers to improve the modularity of a variety of complex gene circuits.

\section{Results and discussion}

\section{A mechanistic model of the resource allocation controller}

We initially develop a complete mechanistic model of gene expression and the action of the controller, before investigating how this model can be simplified for use as a design tool. We assume that each circuit promoter $\left(g_{i}\right)$ can be bound by a multimeric transcription factor $\left(u_{i}\right)$ to form a promoter complex $\left(\kappa_{i}\right)$ capable of recruiting a free RNA polymerase $(\sigma)$ to

form a translation complex. When transcription occurs, an mRNA $\left(m_{i}\right)$ is produced, and 
the original RNAP polymerase and promoter complex are released. The above interactions are described by the following chemical reactions:

$$
g_{i}+\eta_{i} \cdot u_{i} \underset{\alpha_{r_{i}}}{\stackrel{\alpha_{f_{i}}}{\rightleftharpoons}} \kappa_{i} \quad \kappa_{i}+\sigma \underset{\xi_{r_{i}}}{\stackrel{\xi_{f_{i}}}{\rightleftharpoons}} x_{i} \quad x_{i} \stackrel{\tau_{i}}{\longrightarrow} m_{i}+\sigma+\kappa_{i}
$$

The mRNA is bound by a free orthogonal ribosome, $R$, to form a translation complex $\left(c_{i}\right)$. Upon translation, a protein $\left(p_{i}\right)$ is produced and the mRNA and $R$ are released:

$$
m_{i}+R \underset{\beta_{r_{i}}}{\stackrel{\beta_{f_{i}}}{\rightleftharpoons}} c_{i} \quad c_{i} \stackrel{\gamma_{i}}{\longrightarrow} m_{i}+R+p_{i}
$$

Additionally, both mRNAs and proteins degrade at rates $\delta_{m_{i}}$ and $\delta_{p_{i}}$, respectively.

Applying the law of mass action we derive the following ODEs describing the time evolution of the circuit components:

$$
\begin{aligned}
\dot{\kappa}_{i} & =\alpha_{f_{i}} g_{i} u_{i} \eta_{i}-\alpha_{r_{i}} \kappa_{i}-\xi_{f_{i}} \kappa_{i} \sigma+\xi_{r_{i}} x_{i}+\tau_{i} x_{i} \\
\dot{x}_{i} & =\xi_{f_{i}} \kappa_{i} \sigma-\xi_{r_{i}} x_{i}-\tau_{i} x_{i} \\
\dot{m}_{i} & =\tau_{i} x_{i}-\beta_{f_{i}} m_{i} R+\beta_{r_{i}} c_{i}+\gamma_{i} c_{i}-\delta_{m_{i}} m_{i} \\
\dot{c}_{i} & =\beta_{f_{i}} m_{i} R-\beta_{r_{i}} c_{i}-\gamma_{i} c_{i} \\
\dot{p}_{i} & =\gamma_{i} c_{i}-\delta_{p_{i}} p_{i}
\end{aligned}
$$

This represents a simple single-input-single-output (SISO) motif and forms the basis of our model. Complex circuits can be constructed by letting the output from one module form the input to another.

To implement our controller we first consider the conversion of host ribosomes $\left(R_{\text {host }}\right)$ into circuit-specific orthogonal ribosomes $(R)$. The orthogonal 16S rRNA gene promoter $\left(g_{r}\right)$ 
recruits $\sigma$ to form a translation complex $\left(x_{r}\right)$ which produces the orthogonal rRNA $(r)$ :

$$
g_{r}+\sigma \underset{\xi_{r_{r}}}{\stackrel{\xi_{f_{r}}}{\rightleftharpoons}} x_{r} \quad \quad x_{r} \stackrel{\tau_{r}}{\longrightarrow} r+\sigma+g_{r}
$$

The orthogonal 16S rRNA binds host ribosomes, $R_{H}$, and so recruits ribosomes to the circuit-only orthogonal pool, $R$ :

$$
R_{H}+r \underset{\varrho_{f}}{\stackrel{\varrho_{r}}{\rightleftharpoons}} R
$$

In the presence of the controller the orthogonal rRNA gene is regulated by the repressor $p_{f}$. The repressor binds the free $g_{r}$ promoter and prevents the binding of RNA polymerase and associated factors ( $\sigma$ in our model):

$$
g_{r}+\eta_{f} \cdot p_{f} \underset{\alpha_{f_{f}}}{\stackrel{\alpha_{r_{r}}}{\rightleftharpoons}} \kappa_{f}
$$

We model expression of the regulator protein by considering the constitutive expression of its mRNA from an unregulated promoter, $g_{f}$ :

$$
g_{f}+\sigma \underset{\xi_{f_{f}}}{\stackrel{\xi_{r_{f}}}{\rightleftharpoons}} x_{f}
$$

We model the transcription and translation of the repressor's mRNA and protein in the same manner as the circuit genes, as described above. Applying the law of mass action results in 
the following ODEs describing the production of the repressor and intermediate complexes:

$$
\begin{aligned}
& \dot{g}_{f}=-\xi_{f_{f}} g_{f} \sigma+\xi_{r_{f}} x_{f}+\tau_{f} x_{f} \\
& \dot{x}_{f}=\xi_{f_{f}} \kappa_{f} \sigma-\xi_{r_{f}} x_{f}-\tau_{f} x_{f} \\
& \dot{m}_{f}=\tau_{f} x_{f}-\beta_{f_{f}} m_{f} R+\beta_{r_{f}} c_{f}+\gamma_{f} c_{f}-\delta_{m_{f}} m_{f} \\
& \dot{c}_{f}=\beta_{f_{f}} m_{f} R-\beta_{r_{f}} c_{f}-\gamma_{f} c_{f} \\
& \dot{p}_{f}=\gamma_{f} c_{f}-\delta_{p_{f}} p_{f}-\eta_{f} \alpha_{f_{r}} g_{r} p_{f} \eta_{f}+\eta_{f} \alpha_{r_{r}} \kappa_{r}
\end{aligned}
$$

Applying the law of mass action to the o-rRNA promoters and ribosome species yields:

$$
\begin{aligned}
\dot{g}_{r} & =-\xi_{f_{r}} g_{r} \sigma+\xi_{r_{r}} x_{r}+\tau_{r} x_{r}-\alpha_{f_{r}} g_{r} p_{f}^{\eta_{f}}+\alpha_{r_{r}} \kappa_{r} \\
\dot{x}_{r} & =\xi_{f_{r}} g_{r} \sigma-\xi_{r_{r}} x_{r}-\tau_{r} x_{r} \\
\dot{\kappa}_{r} & =\alpha_{f_{r}} g_{r} p_{f}^{\eta_{f}}+\alpha_{r_{r}} \kappa_{r} \\
\dot{r} & =\tau_{r} x_{r}-\delta_{r} r-\varrho_{f} r R_{H}+\varrho_{r} R \\
\dot{R}_{H} & =-\varrho_{f} r R_{H}+\varrho_{r} R
\end{aligned}
$$

This detailed model is highly complex; being composed of 5 species per circuit gene and the 10 species of the controller. Additionally, some parameters, such as on and off rates, are difficult to determine experimentally and so they are unlikely to be available in the literature preventing design. We reduce this model by considering the effect of time-scale separation and species conservation (see Section S1). Additionally, we remove the potentially confounding RNA polymerase mediated coupling as discussed in Section S1. The tractable model is composed of 3 ODEs describing the action of the controller and one equation per 
circuit protein:

$$
\begin{aligned}
\dot{p}_{i} & =\gamma_{i} \hat{c}_{i} R-\delta_{p_{i}} p_{i} \\
\dot{p}_{f} & =\gamma_{f} \hat{c}_{f} R-\delta_{p_{f}}-\eta_{f} \bar{g}_{r} p_{f} \eta_{f}+\eta_{f} \mu_{f} \bar{\kappa}_{r} \\
\dot{r} & =\tau_{r} \bar{x}_{r}-\delta_{r} r-\varrho_{f} r R_{h}+\varrho_{r} R \\
\dot{R}_{h} & =-\varrho_{f} r R_{h}+\varrho_{r} R
\end{aligned}
$$

where $\hat{c}$ is a measure of the demand for ribosomes and includes the quasi-steady state transcriptional dynamics. $\hat{c}$ is defined fully in Equation S10. $\bar{g}_{r}$ and $\bar{\kappa}_{r}$ are the quasi-steady state free and inhibited o-rRNA promoter concentrations respectively (Equations S12 and S14). The concentration of free orthogonal ribosomes is given by:

$$
R=\frac{R_{\text {Total }}-R_{h}}{1+\hat{c}_{f}+\Sigma_{1}^{N}\left(\hat{c}_{i}\right)}
$$

Note that this follows the same form as Equation 2, with the total number of o-ribosomes available to the circuit being the total number of all ribosomes $\left(R_{\text {Total }}\right)$ minus the number of host ribosomes $\left(R_{\text {host }}\right)$. (Note that the majority of this work is concerned with two gene circuits and therefore $N=2$.)

\section{Model analysis reveals a trade off between gene expression and level of decoupling}

We considered a simple circuit consisting of two modules single-input-single-output motifs. We assess the impact of the controller to reject disturbances to one module upon activation of the second: i.e. we assess the change in $p_{1}$ upon activation of $u_{2}$. This difference in expression of $p_{1}$ due to the $u_{2}$ disturbance is termed 'coupling' below (see Methods for quantification). We assess any impact on protein levels by comparing the final protein

outputs to the theoretical maximum: i.e. the protein levels obtained using the host ribosome 
pool for translation and assuming maximal induction. We initially consider how tuning the circuit inputs $\left(u_{1}\right.$ and $u_{2}$ ) only impacts gene expression and coupling in a circuit translated by the host ribosome pool (and therefore in the absence of feedback control). This demonstrates a linear trade-off with coupling falling as expression falls. Using the reduced five state model to design our feedback controller, we carried out a multiobjective optimisation of the experimentally tunable controller parameters aiming to produce both high protein levels and low gene coupling (see Methods). We sampled parameters around the front to identify the true shape of the Pareto-optimal front, corresponding to a controller co-operativity $\eta_{f}$ of 4. This identified a hard trade-off between these two objectives, with the range of equally optimal solutions showing an inverted concave shape, i.e. decreases in gene coupling are achieved at the expense of decreases in gene expression (Figure 1c). Our simulations suggest that the controller can halve coupling for only a 13\% reduction in expression (for the highest co-opperativity $\eta_{f}=4$ ); in comparison the tuning circuit inputs requires a $48 \%$ reduction in expression to achieve the same level of coupling. If a loss of $\sim 50 \%$ is acceptable then coupling can be reduced to only $5 \%$. The controllers give access to a higher level of gene expression for a given level of decoupling than tuning circuit parameters alone.

We demonstrate the functioning of the controller using Point 9 as determined by our optimisation routine. This controller designs shows intermediate results with coupling falling to only $5 \%$ for a loss of $50 \%$ expression. The corresponding open loop arrangement where the inputs $u_{1}$ and $u_{2}$ tuned to produce the same final expression shows coupling of $15 \%$. The controller successfully insulates one gene from the induction of another (Figure 1d), bar a short transient disturbance $(<12 \mathrm{~h})$. Tracking the concentrations of the intermediate species reveals the operation of the controller; with translation of $p_{f}$ falling and the number of o-rRNA genes being transcribed increasing as the second gene is induced (Figure 1e). This results in a net increase in the number of orthogonal ribosomes (Figure 1f) which means that in the long term the translation complexes producing each protein do not change (Figure 1d). 
To identify the true Pareto front and determine the robustness of the trade-off we varied the results of our genetic algorithm by up to $50 \%$ for each point identified. This identifies the true Pareto front when $\eta_{f}=4$. None of the designs from this robustness analysis falls below the linear trade-off achieved by input tuning; i.e. there are no designs where the action of the controller reduces expression without having a beneficial effect on gene coupling. A number of controllers provide no improvement upon input tuning (see below). We find that a small number of these perturbed designs show slower responses and we discount these from further analysis. We carried out an additional robustness analysis allowing all parameters governing the controller behaviour to vary. This includes parameters which are either difficult to design (e.g. controller translation rate $\gamma_{f}$ ) or intrinsic properties which cannot be designed (e.g. o-rRNA association rate, $\mu_{r}$ ). All of these controllers also fall upon the same front demonstrating that uncertainty in these values does not preclude controller design (Figure S1).

To determine how each parameter contributes to the gene expression and coupling trade off, we analysed how each changes across the front. This highlights the need for high $\eta_{f}$ values. This parameter represents the level of co-operativity in the system brought about, for example, by transcription factor multimerisation or the presences of multiple operator sites. The true Pareto front coincides with a value of $\eta_{f}=4$ (Figure 2b). Due to the present constraint that most repressors used in synthetic biology are derived from natural sources and therefore it is unlikely that all combinations of multimerisation $\left(\eta_{f}\right)$, dissociation constant $\left(\mu_{f}\right)$ and promoter kinetics $\left(k_{X_{r}}\right)$ desired will be present in one selected repressor and so we also consider designs based on suboptimal dimeric and trimeric repressors $\left(\eta_{f}=2\right.$ and 3). However, given the poor performance of monomeric controllers $\left(\eta_{f}=1\right)$ we discount these from all further analysis. Across all $\eta_{f}$ values, small $\mu_{f}$ values are most often associated with controllers which act to nearly completely decouple genes but at a significant cost to gene expression (Figure 2d) with only $\mu_{f}>1 \mathrm{nM}$ giving access to moderately high levels of expression. Similarly, small $k_{L_{f}}$ values, corresponding to strong ribosomes binding sites (low 
ribosome-mRNA dissociation constant), are associated with large levels of decoupling at a high cost to gene expression (Figure 2c). Simulations suggest $k_{L_{f}}>10^{5} \mathrm{nM}$ and $\mu_{f}>0.1 \mathrm{nM}$ in all cases, for the simple two gene circuit example used here. (Note that for many natural transcription factors co-opted into synthetic gene networks $\mu_{f}<0.1 \mathrm{nM}$ and $\eta_{f}$ may be limited. We demonstrate how this can be compensated for in controller design in Section 2.5). A high $g_{f, T} / k_{X_{f}}$ ratio $\left(g_{f, T} / k_{X_{f}}>1\right.$, produced by expressing the regulator from a strong promoter carried on a high copy number plasmid) results in complete decoupling and abolition of gene expression (Figure 2b). We therefore suggest keeping $g_{f, T} / k_{X_{f}}<1$ in all instances. We find that the $g_{r, T} / k_{X_{r}}$ ratio governing maximal o-rRNA transcription rate varies significantly across all behaviours making general guidelines difficult to establish (Figure 2a).

To provide further specific quantitative design rules which manage the trade-off, we divided the responses of the different controllers based on the their decoupling ability and final circuit expression level (Figure 2e). These represent groups of controllers with similar behaviours, see Methods. This shows that the key determinant of the expression-coupling trade-off is the RBS strength $\left(k_{L_{f}}\right)$ with the dissociation constant decreasing with increased decoupling (Figure 2e, inset). (Note that for the majority of behaviours $k_{L_{f}}$ is greater than $10^{8} \mathrm{nM}$. This represents a weaker RBS strength than currently available requiring extensive RBS engineering.) The o-rRNA transcriptional parameters $\left(g_{r, T} / k_{X_{r}}\right.$ ratio $)$ is between 0.1 and 1 which can be achieved through a range of different plasmid copy number and promoter dissociation constants (2e, inset. Figure S4. The gene encoding the controller protein should be expressed from a weak promoter and a low or medium copy plasmid in order to create a $g_{f, T} / k_{X_{f}}$ ratio of between 0.01 and 0.1 (Figure 2e, inset). The controller dissociation constant $\mu_{f}$ can take any value between $10 \mathrm{nM}$ and $\sim 30 \mathrm{nM}$. For the most strongly decoupling controllers a greater range of $g_{f, T} / k_{X_{f}}$ and $\mu_{f}$ are tolerated; with the greater range corresponding to a higher expression of controller and stronger repressor binding. The same patterns are observed across $\eta_{f}=2$ and 3 (Figure S2). 


\section{Designing system response times by tuning controller parameters}

Analysis of the controllers tested so far has focused on how they are able to correct steady state errors brought about by gene coupling and so we have largely ignored the system dynamics, bar excluding excessively slow controllers (e.g. penalising simulations which only reach steady state after $>24 \mathrm{~h}$ ). However, a controller which decouples genes well but has a slow response time will not be suitable for many applications in synthetic biology. Therefore we took the previous candidate controllers and conducted a local sensitivity analysis around each design point to assess the impact of each parameter on the system's speed of response. In addition to the controller parameters varied so far we also varied $\delta_{\rho}, \delta_{m_{f}}$ and $\delta_{p_{f}}$, which represent the decay of the o-rRNA, controller mRNA and controller protein respectively. These parameters were kept constant in the previous design evaluations to minimise the number of parameters in the optimisation, but since decay parameters often have significant affects on speed of response we explicitly assess their impact here.

The o-rRNA decay constant $\left(\delta_{r}\right)$ and protein controller decay constant $\left(\delta_{p_{f}}\right)$ are key to determining the speed of the system response. Increasing both parameters acts to increase the speed of response (Figure 3). Increasing $\delta_{r}$ increases the controllers ability to decouple circuit genes. Increasing $\delta_{p_{f}}$ decreases decoupling ability but increases expression. In the regions tested, varying $\delta_{r}$ is less likely to introduce significant overshoots into the system (as seen at low $\delta_{p_{f}}$ values). However, a greater range of speed-up is achievable by varying the protein decay constant. The latter is also a more experimentally tractable parameter. Increasing both parameters acts antagonistically, with increases in $\delta_{r}$ decreasing gene coupling and increases in $\delta_{p_{f}}$ increasing it, meaning tuning both parameters may be advantageous. We see very little impact from varying the mRNA decay rate $\left(\delta_{m_{f}}\right)$. As previously discussed the value of the controller co-operativity $\left(\eta_{f}\right)$ is a key determinant of controller decoupling ability. This analysis replicates this result and also highlights that, at least in this parameter regime, increasing co-operativity also acts to significantly increase the speed of response (Figure 3). 
Given that changing these three parameters significantly changes the decoupling ability of the controller in addition to its dynamics, we assessed the impact of varying additional parameters to design controllers with increased response times while maintaining controller function (as measured by decoupling and expression). The changes in decoupling ability brought about by increasing $\delta_{r}$ can be ameliorated by decreasing the expression of the controller protein through tuning $g_{f, T} / k_{X_{f}}$ (Figure $3 \mathrm{~g}$ ). Whilst in Figure $3 \mathrm{~g}$, the time it takes for $p_{1}$ to return to steady state after induction of $p_{2}$ at $12 \mathrm{~h}$ does not change the $p_{2}$ rise time (i.e. the time it takes for $P_{2}$ to rise from $10 \%$ to $90 \%$ of its final value) falls by $30 \%$. The loss in decoupling ability associated with increasing the controller protein decay rate can likewise be corrected by increasing $g_{f, T} / k_{X_{f}}$ (Figure $3 \mathrm{~h}$ ). In Figure 3i, the dynamics are significantly improved by increasing $\eta_{f}$ from 2 to 4 with the $p_{1}$ settling time falling by 5 hours. To maintain expression levels $\mu_{f}$ of the controller protein needs to be tuned; but this may not be possible given the reuse of natural components as discussed above.

\section{Potential biological implementations of the controller designs}

We carried out a detailed literature review to identify potentially suitable repressors with which to implement our system, focusing our analysis on (i) the ability of the repressor to be expressed in bacterial hosts (i.e. repressors from bacteria or bacteriophage), (ii) orthogonality (i.e. repressors which are not used in fundamental host processes), (iii) the presence of a known promoter architecture (which could be used to infer the dissociation constant of the RNA polymerase, see Section S2) and (iv) detailed characterisation of binding kinetics (ideally dissociation constants measured in a biochemical assay, rather than a constant inferred from device function such as by fitting a Hill function to induction-fluorescence curves, as is often the case). We identified six repressors from this literature search, including the commonly used LacI, ${ }^{18}$ TetR $^{19}$ and $\mathrm{cI}^{20}$ repressors. We also identified putative controller candidates Cro and RstR from bacteriophages PY54, ${ }^{21} \mathrm{CTX} \varphi^{22}$ and LmrR, a global regulator of antibiotic resistance from Gram positive Lactococcus lactis ${ }^{23}$. 
Using the results of our sensitivity analysis and additional biological constraints we identified a number of feasible biological implementations. We considered two gene expression systems; (i) where the o-rRNA and regulator having the same medium copy number (mimicking placement in the same plasmid, such as ColE1) and (ii) a high copy number o-rRNA gene, carried on for example a pUC vector, and a chromosomally integrated regulator. Note that we did not assess the potential designs requiring the o-rRNA and regulator to be carried on different copy number plasmids, as these would result in high burden on the cells and significantly decreased growth rate as these cells would need to carry at least three plasmids, one containing circuit genes and one each for the o-rRNA gene and regulator. We explored the potential impact of engineering the o-rRNA promoter strength by multiplying the reported dissociation constant (as reported in the literature or calculated see Section S2) by 0.25 (representing a four-fold strengthening of the promoter) and 2 (representing a two fold weakening of the promoter). We simulated three different controller promoter strengths representing strong $(200 \mathrm{nM})$, medium $(500 \mathrm{nM})$ and weak (1000 nM) promoters. We also assessed the impact of varying RBS strength. We also assessed the impact of fusing degradation tags to the controller protein. The small amino acid motifs acts to target proteins for degradation and hence increase $\delta_{p_{f}}$. These simulations of potential controller designs shows that by selecting different biological components all levels of the trade-off are accessible. (Figure 4).

These different implementations have a wide range of dynamics. Selecting those controllers with high decoupling ability (i.e. where coupling was greater than -0.15), we ranked them by the length of time it took $p_{1}$ to return to steady state after the disturbance caused by $u_{2}$ and selected the fastest responding implementation for each repressor (Figure 5). See Table S5 for details of the designs shown. All these implementations have similar $p_{2}$ rise times of approximately 3 hours and show similar disturbances to $p_{1}$ upon induction of $u_{2}$ (Figure 5, e). The putative controllers based on the tetramers LacI and RstR show the fastest respond times with $p_{1}$ settling with 10 hours of $p_{2}$ induction (Figure $5 \mathrm{~b}$ ). The designs 
based on dimers show the greater outputs, with a concurrent increase in coupling between 7 and $14 \%$ depending upon the controller design (Figure 5c).

\section{A dynamic resource allocation controller restores modularity in a range of more complex gene circuits}

Having successfully demonstrated the ability of the proposed approach to decouple two independent modules, we analyse the ability of the controller to remove resource dependent failure in a variety of more complex gene circuits (Figure 6).

We initially simulate multiple SISO modules with new modules being activated at different intervals. In the absence of the controller, activation of each additional module has an impact on the previously activated modules. For example, the expression of the first module $p_{1}$ falls by over $50 \%$ as three additional genes are induced. As shown in Figure 6a, the controller successfully eliminates this coupling, making $p_{1}$ relatively insensitive to the induction of over 10 additional genes (although note that the rise time and settling time increase slightly with the induction of each additional gene).

A key aim of synthetic biology is that previously characterised components or devices can be introduced into the same cell to form a complex circuit. Here we assess the effect of introducing two separately characterised devices into one complex circuit, i.e. we want to investigate what is the effect of introducing an additional resource consumer on a previously characterised device. As the production of robust genetic oscillators to create clocks for temporal processes functions is of fundamental importance in synthetic circuit design, we consider designs for the repressilator clock and an additional SISO module. These modules are first simulated separately, as shown in (Figure 6b, upper panel). Upon linking these separate devices through a common pool of resources, i.e. coupled through their competition for ribosomes, we see that $p_{4}$ induction destroys the oscillations of the repressilator (Figure $6 \mathrm{~b}$, upper right panel). If, however, we consider the design of these two devices in the presence of the controller and then introduce them into the same resource pool as before, 
we see that circuit function is now maintained (Figure 6b, lower panels). Note that while there is still a small loss in repressilator amplitude upon induction of $p_{4}$ this is significantly reduced, thus staying closer to the original device behaviour.

It has previously been shown that resource limitations can change the input-output response of a simple genetic activation cascade $^{4}$. The authors show that if the upstream module has a stronger ability to sequester ribosomes than the downstream module (a small $k_{L_{1}}$-to- $k_{L_{2}}$ ratio) then the expected response determined from simple Hill-function type modelling (i.e. an increasing output to increasing input in a step-like fashion) can become biphasic or even invert (Figure 6c, dotted open loop lines). We simulate a range of prototype activation cascades in the absence and presence of our controller. In the absence of the controller, no additional resources are available as demand increases and so we see the activation cascade failing in the same manner as found in Qian et al.. In the presence of the controller, the desired behaviour of the activation cascade is restored, as translational capacity is directed to the circuit as demand increases. The controller acts to remove the resource limitation, thus allowing simpler models, which often do not account for limited cellular resources, to be used to produce circuit designs which then function as expected in vivo.

\section{Conclusions}

Numerous genetic components and devices have been developed to ensure predictable gene expression or dampen the effect of loading in genetic circuits. However, to date, little attention has been paid to developing genetic devices that are capable of relieving cellular resource limitations. Controllers for orthogonal transcriptional activity based on phage RNA polymerases have been developed ${ }^{25,26}$ and we have previously implemented a prototype translational controller ${ }^{14}$. Here, we develop a detailed mechanistic model of gene expression and resource allocation, which when simplified to a tractable level of complexity, allows the rational design of optimal translational controllers. We demonstrated that this new model allows the design of controllers which can dynamically allocate orthogonal ribosomes to syn- 
thetic circuits within reasonable timeframes ( $<12$ hours). Using our model, we identify a fundamental trade-off in controller design; that reducing coupling act to decrease gene expression. We determined how each controller design parameter affects the overall closedloop behaviour of the system, leading to a detailed set of design guidelines for optimally managing this trade-off. We find that both controller co-operativity and RBS strength are key parameters in determining the level of decoupling that can be achieved. Based on our designs, we identified and evaluated a number of alternative potential experimental implementations of the proposed system using commonly available biological components. Finally, we showed that our controller is capable of dynamically allocating ribosomes as needed to restore modularity in a number of more complex synthetic circuits, such as the repressilator, and activation cascades composed of multiple interacting modules.

\section{Methods}

\section{Numerical simulations}

All models were implemented in either MATLAB 2016b and 2017a (The MathWorks Inc, MA, USA) and simulated using the in-built stiff solvers ode15s and ode23s using increased tolerances (Relative $10^{-6}$ and Absolute $10^{-6}$ ). Simulations were deemed to have reached steady state when the maximum of the calculated derivative was less than $10^{-2}$ (relaxed) or $10^{-3}$ (strict). Additional specialist functions as needed were utilised from the Optimization Toolbox (Version 7.4) and Parallel Computing Toolbox (Version 6.8 or 6.10).

\section{Assessment of controller function}

The behaviour of controllers was characterised by simulating the action of a simple two gene circuit. Initially, the behaviour of one gene $p_{1}$ is simulated before its response is assessed to 
the induction of a second gene $p_{2}$ at time $t=\theta_{\text {ind }}$. Coupling and expression are normalised:

$$
\begin{aligned}
\text { coupling } & =\left(p_{1}\left(t=\theta_{\text {ind }}\right)-p_{1}\left(t=\theta_{s s}\right)\right) / p_{1}\left(t=\theta_{\text {ind }}\right) \\
\text { expression } & =\left(p_{2}\left(t=\theta_{\text {ss }}\right)-p_{\text {target }}\right) / p_{\text {target }}
\end{aligned}
$$

We calculate the fold reduction in expression by taking:

$$
\text { fold reduction in expression }=\frac{1}{1-\mid \text { expression } \mid}
$$

\section{Optimisation}

The mutliobjective optimisation was carried out using the inbuilt function gamuliobj with a population size of 200 individuals and with a Pareto fraction of 0.25 from the Optimization Toolbox. $k_{X}$ values were set to 1 allowing the $\hat{x}$ ratios to be investigated by varying $g_{r, T}$ and $g_{f, T}$ only. See Section S2 for a discussion of permissible parameter bounds. The parameters varied (and their scale and bounds) were $g_{r, T} / k_{X_{r}}$ ratio $\left(\log 10\right.$ scale, $\left.10^{-2}-10^{2}\right), k_{L_{f}}(\log 10$ scale, $10^{3}-10^{8}$ ), $\eta_{f}$ (integers values only to represent the number of required monomers in the DNA binding complex, $1-4), \mu_{f}\left(\log 10\right.$ scale, $\left.10^{-2}-10^{3}\right)$ and $g_{r, T} / k_{X_{r}}$ ratio $(\log 10$ scale, $10^{-2}-10^{2}$ ). The optimisation routine aims to minimise:

$$
\begin{aligned}
& \chi_{1}=\left(p_{1}\left(t=\theta_{\text {ss }}\right)-p_{1}\left(t=\theta_{\text {tind }}\right)\right)^{2} \\
& \chi_{2}=\left(p_{2}\left(t=\theta_{\text {ss }}\right)-p_{\text {target }}\right)^{2}
\end{aligned}
$$

where $\theta_{\text {ind }}$ is the time of the induction of $p_{2}$ and $\theta_{s s}$ is the last time point, $t_{\max }$. If the simulation is not at steady state at $t_{\max }$ then the result is given the poorest fitness. $p_{\text {target }}$ is calculated by simulating the action of the circuit in a model utilising the host ribosome pool for gene expression. 


\section{Selection of controller parameters for design guidelines}

Coupling and expression scores where calculated for each controller as outlined above for all the results of robustness analysis. These results were then scaled by their maximum absolute values to ensure both axes are between 0 and 1 (note that for calculating the distance metric we can ignore signs):

$$
x_{\text {scaled }}=1+\frac{\text { coupling }}{\max (\mid \text { coupling } \mid)} \quad y_{\text {scaled }}=1+\frac{\text { expression }}{\max (\mid \text { expression } \mid)}
$$

Given the curved nature of the Pareto front we recast the Euclidean coordinates into polar coordinates:

$$
r=\sqrt{\left(x_{\text {scaled }}^{2}+y_{\text {scaled }}^{2}\right)} \quad \theta=\arccos \left(x_{\text {scaled }} / r\right)
$$

We divide the Pareto front into five segments ('behaviours') by the corresponding angle $\theta$ using the inbuilt MATLAB function discretize. This determines parameter regimes for the given behaviour.

\section{Acknowledgement}

APSD acknowledges funding from the University of Warwick and the EPRSC \& BBSRC Centre for Doctoral Training in Synthetic Biology (grant EP/L01649/1). High performance computational facilities were provided by Warwick Integrative Synthetic Biology Centre, funded by BBSRC/EPSRC (grant BB/M017982/1). JK and JIJ acknowledge support from the European Union's Horizon 2020 research and innovation programme (grant no. 633962) and support from the BBSRC (grant BB/M009769/1). 


\section{Supporting Information Available}

The following files are available free of charge.

- Supplementary Material: Supplementary Figures, Model reduction, Discussion of parameterisation, Circuit models. 


\section{References}

1. J. A. Brophy and C. A. Voigt, "Principles of genetic circuit design.," Nat. Methods, vol. 11, no. 5, pp. 508-20, 2014.

2. S. Cardinale and A. P. Arkin, "Contextualizing context for synthetic biology - identifying causes of failure of synthetic biological systems," Biotechnol. J., vol. 7, no. 7, pp. 856-866, 2012.

3. D. Del Vecchio, A. J. Ninfa, and E. D. Sontag, "Modular cell biology: retroactivity and insulation.," Mol. Syst. Biol., vol. 4, no. 161, p. 161, 2008.

4. Y. Qian, H.-H. Huang, J. Jiménez, and D. Del Vecchio, "Resource competition shapes the response of genetic circuits," ACS Synth. Biol., vol. 6, no. 7, pp. 1263-1272, 2017.

5. C. Lou, B. Stanton, Y.-J. Chen, B. Munsky, and C. A. Voigt, "Ribozyme-based insulator parts buffer synthetic circuits from genetic context.," Nat. Biotechnol., vol. 30, no. 11, pp. 1137-42, 2012.

6. C. J. Bashor and J. J. Collins, "Insulating gene circuits from context by RNA processing," Nat. Biotechnol., vol. 30, no. 11, pp. 1061-1062, 2012.

7. K. S. Nilgiriwala, J. Jimenez, P. M. Rivera, D. D. Vecchio, J. Jiménez, P. M. Rivera, and D. Del Vecchio, "Synthetic tunable amplifying buffer circuit in E. coli," ACS Synth. Biol., vol. 4, pp. 577-84, 2015.

8. M. Scott, C. W. Gunderson, E. M. Mateescu, Z. Zhang, and T. Hwa, "Interdependence of cell growth and gene expression: origins and consequences," Science, vol. 330, no. 6007, pp. 1099-1102, 2010.

9. F. Ceroni, R. Algar, G.-B. Stan, and T. Ellis, "Quantifying cellular capacity identifies gene expression designs with reduced burden," Nat. Methods, vol. 12, no. 5, pp. 415-423, 2015. 
10. A. Gyorgy, J. I. Jiménez, J. Yazbek, H.-H. Huang, H. Chung, R. Weiss, and D. Del Vecchio, "Isocost lines describe the cellular economy of genetic circuits," Biophys. J., vol. 109, no. 3, pp. 639-646, 2015.

11. M. Carbonell-Ballestero, E. Garcia-Ramallo, R. Montanez, C. Rodriguez-Caso, and J. Marcia, "Dealing with the genetic load in bacterial synthetic biology circuits : convergences with the Ohm's law," Nucleic Acids Res., vol. 44, no. 1, pp. 496-507, 2016.

12. T. E. Gorochowski, I. Avcilar-Kucukgoze, R. A. Bovenberg, J. A. Roubos, and Z. Ignatova, "A minimal model of ribosome allocation dynamics captures trade-offs in expression between endogenous and synthetic genes," ACS Synth. Biol., vol. 5, pp. 710-720, 2016.

13. T. Shopera, L. He, T. Oyetunde, Y. J. Tang, and T. S. Moon, "Decoupling resourcecoupled gene expression in living cells," ACS Synth. Biol., vol. 6, no. 8, pp. 1596-1604, 2017.

14. A. P. S. Darlington, J. Kim, J. I. Jimenez, and D. G. Bates, "Dynamic allocation of orthogonal ribosomes facilitates uncoupling Of co-expressed genes," Nat. Commun., vol. 9, pp. 695. 2018.

15. R. Milo and R. Phillips, Cell Biology by the numbers. New York, NY: Garland Science, 2016.

16. H. Bremer, P. Dennis, and M. Ehrenberg, "Free RNA polymerase and modeling global transcription in Escherichia coli," Biochimie, vol. 85, no. 6, pp. 597-609, 2003.

17. A. Hamadeh and D. del Vecchio, "Mitigation of resource competition in synthetic genetic circuits through feedback regulation," 53rd IEEE Conference on Decision and Control, Los Angeles, CA, USA. pp. 3829-3834, 15-17 December 2014.

18. C. M. Falcon and K. S. Matthews, "Operator DNA sequence variation enhances high 
affinity binding by hinge helix mutants of lactose repressor protein," Biochemistry, vol. 39, no. 36, pp. 11074-11083, 2000.

19. A. Kamionka, J. Bogdanska-Urbaniak, O. Scholz, and W. Hillen, "Two mutations in the tetracycline repressor change the inducer anhydrotetracycline to a corepressor," Nucleic Acids Res., vol. 32, no. 2, pp. 842-847, 2004.

20. Y. Wang, L. Guo, I. Golding, E. C. Cox, and N. P. Ong, "Quantitative transcription factor binding kinetics at the single-molecule level," Biophysical J., vol. 96, no. 2, pp. 609620,2009 .

21. J. A. Hammerl, N. Roschanski, R. Lurz, R. Johne, E. Lanka, and S. Hertwig, "The molecular switch of telomere phages: High binding specificity of the PY54 Cro lytic repressor to a single operator site," Viruses, vol. 7, no. 6, pp. 2771-2793, 2015.

22. H. H. Kimsey and M. K. Waldor, "The CTX $\phi$ repressor RstR binds DNA cooperatively to form tetrameric repressor-operator complexes," J. Biol. Chem., vol. 279, no. 4, pp. 26402647, 2004.

23. H. Agustiandari, E. Peeters, J. G. de Wit, D. Charlier, and A. J. M. Driessen, "LmrRmediated gene regulation of multidrug resistance in Lactococcus lactis," Microbiology, vol. 157, no. 5, pp. 1519-1530, 2011.

24. O. Purcell, C. S. Grierson, M. di Bernardo, and N. J. Savery, "Temperature dependence of ssrA-tag mediated protein degradation," J. Biol. Eng., vol. 6, no. 1, pp. 10, 2012.

25. T. H. Segall-Shapiro, A. J. Meyer, A. D. Ellington, E. D. Sontag, and C. A. Voigt, "A 'resource allocator' for transcription based on a highly fragmented T7 RNA polymerase.," Mol. Syst. Biol., vol. 10, no. 7, pp. 742, 2014.

26. M. Kushwaha and H. M. Salis, "A portable expression resource for engineering crossspecies genetic circuits and pathways," Nat. Commun., vol. 6, pp. 7832, 2015. 
a
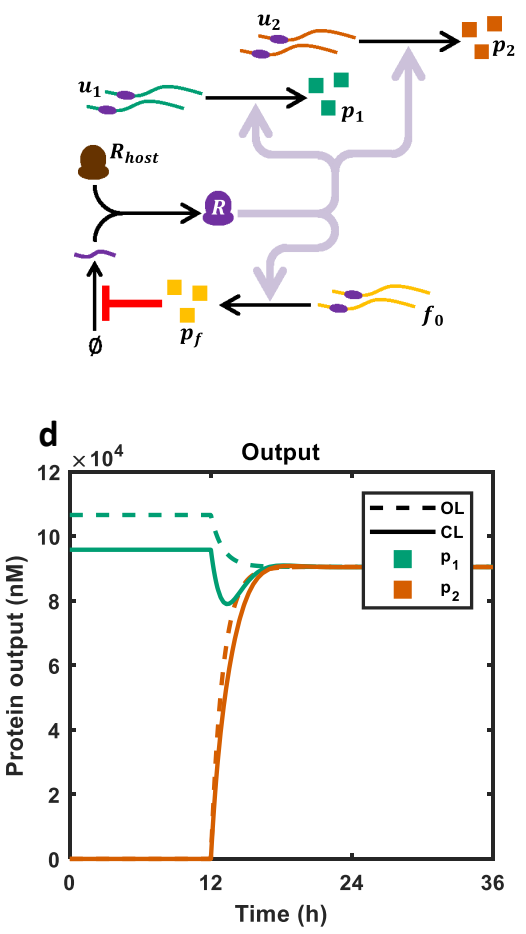

b
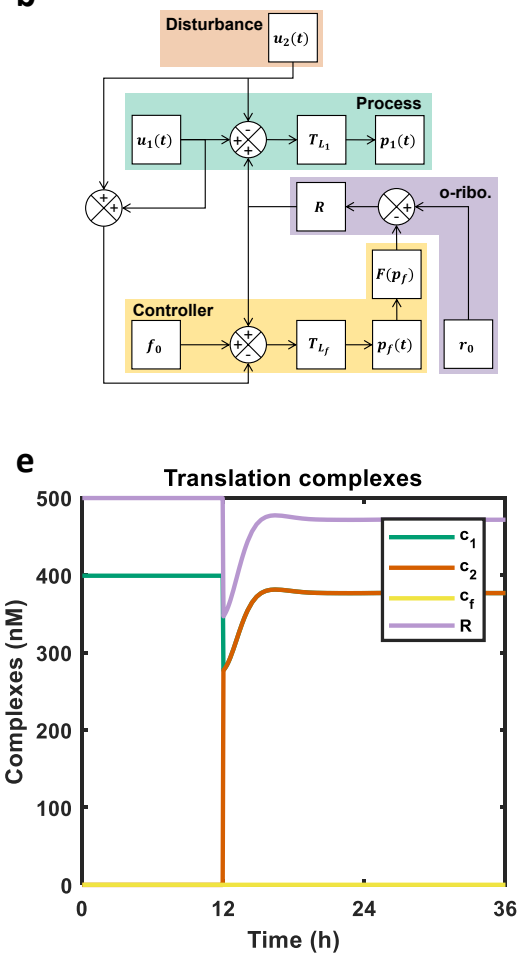

C

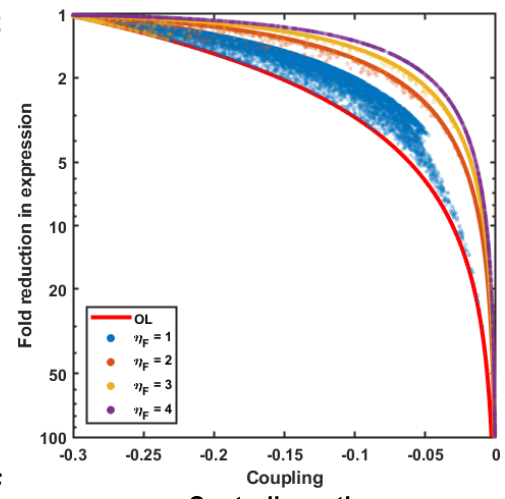

f

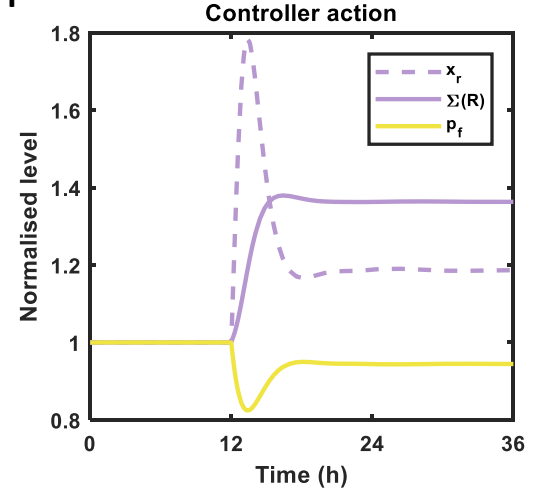

Figure 1: Development of a genetic feedback controller model (a) Schematic of the negative feedback loop implementation. (b) Block diagram of the controller. The process, highlighted in green, converts the input $u_{1}$ into protein output $p_{1}$ utilising the o-ribosome pool $R$. The input into a second process (not shown) $u_{2}$ acts as a disturbance to the first process which is ameliorated by the effect of the controller. The controller protein is constitutively expressed ( $f_{0}$ signal) so the output $p_{f}$ is dependent upon $R$. As inputs $u_{i}$ disturb $R$ the level of $p_{f}$ changes (i.e. as $u_{i}$ increases, $p_{f}$ decreases). As $p_{f}$ is a repressor the disturbance signal is inverted in the $F\left(p_{f}\right)$ block. (c) Gene expression and decoupling trade-off. Expression and coupling were calculated as defined in the Methods. The results of the multiobjective optimisation where perturbed by $\pm 50 \%$ to discover the shape of the true Pareto front. The controller results are divided by $\eta_{f} . N=107,743$. OL, open loop trade-off determined by varying the values of the inputs for a circuit using the host ribosome pool, which by definition is uncontrolled. (d)-(f) Controller dynamics as it decouples circuit genes using the parameters of Point 19 of Table S1. The first gene $p_{1}$ is constitutively expressed throughout, $u_{1}=500 \mathrm{nM}$ throughout. At $12 \mathrm{~h}, u_{2}$ rises from 0 to $500 \mathrm{nM}$. (d) Protein output of the close loop system. OL represents the coupling when the inputs are tuned in the absence of the controller to match the final protein level. (e) Translation complexes: Changing distributions of orthogonal ribosomes across circuit and controller mRNAs. $c_{1}, c_{2}$ and $c_{f}$ represent the translation complexes of the mRNAs of genes 1, 2 and the regulator $f$ respectively. $R$ represents the free orthogonal ribosomes. $c_{f}$ acts as the sensor for the disturbance at $\mathrm{t}=12 \mathrm{~h}$. Levels are a normalised by the total number of orthogonal ribosomes at $\mathrm{t}=12 \mathrm{~h}$. (f) Controller action: Changes in controller components over time. Levels are normalised by value at $\mathrm{t}=12 . x_{r}$, o-16S rRNA gene in the transcribing state; $\Sigma(R)$, number of orthogonal ribosomes; $p_{f}$, controller protein. 

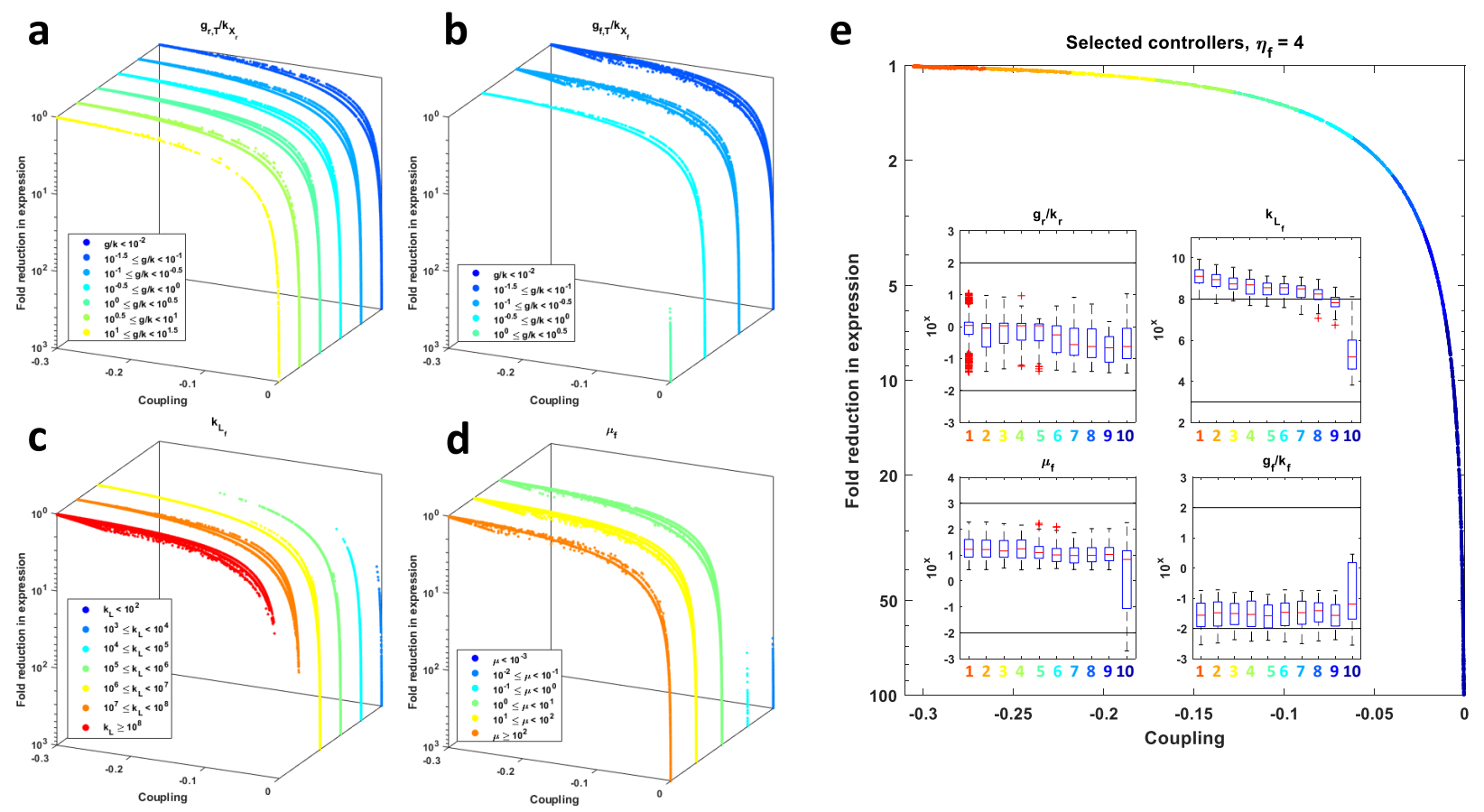

Figure 2: Design trade-offs between gene expression and decoupling. Designs of controllers which manage the trade-off between gene expression and decoupling. Expression and coupling are calcaulted as defined in the Methods. $N=107,743$. As described in the main text, controllers where $\eta_{f}=1$ are removed from the following panels for clarity. Also note that the third axis in plots (a) to (d) and subsequent separation serves only to aid visualisation and does not represent parameter value which is indicated by the colour as outlined in the figure legend. Points with greater fold reduction than $10^{3}$ are not shown. All these points represent complete decoupling. (a) o-rRNA transcription as determined by the $g_{r, T} / k_{X_{r}}$ ratio. (b) Transcription of the controller protein as determined by the $g_{f, T} / k_{X_{f}}$ ratio. (c) Controller mRNA ribosome binding site strength as measure by mRNAribosome dissociation constant $k_{L_{f}}$. (d) Controller protein $g_{r}$ dissociation constant $\mu_{f}$. (e) Parameter changes across the Pareto front (i.e. controllers where $\eta_{f}=4$ ). The different trade-offs between coupling and expression are divided into similarly behaving controllers (as described in the Methods). Insets, show the median parameter value and interquartile range for each set of behaviours. 

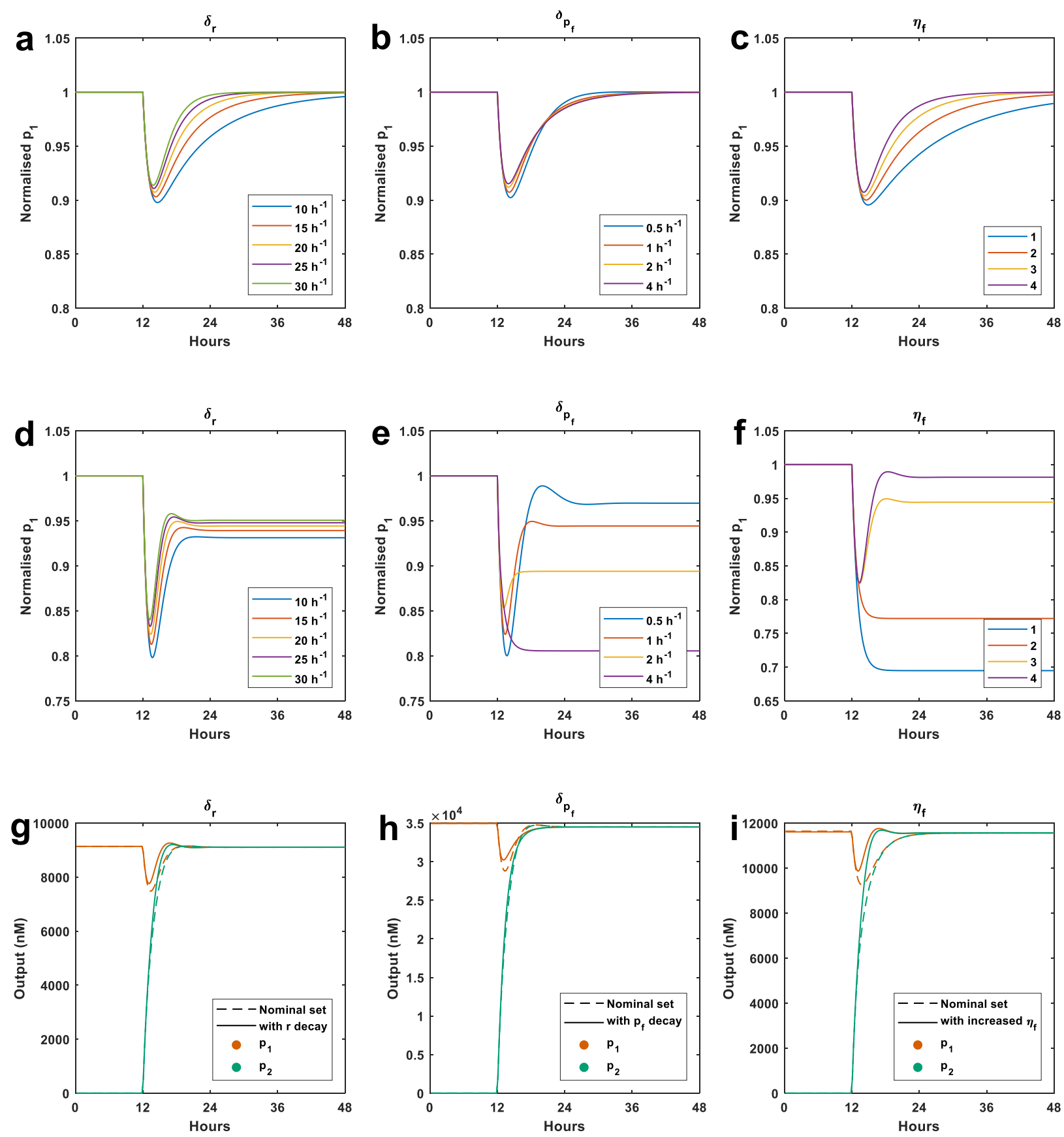

Figure 3: Tuning decay parameters allows design of system dynamics. The effect of varying the decay prameters $\delta_{r}$ and $\delta_{p_{f}}$ and the co-operativity parameter $\eta_{f}$ on the response of $p_{1}$ to the additional input $u_{2}$. Panels (a), (b) and (c) represents the results of the sensitivity analysis around the parameter set from the high decoupling regime (Point 1 of Table S1). Panels (d), (e) and (f) represents the results of the sensitivity analysis around a parameter set from the intermediate decoupling regime (Point 42 of Table S1). In Panels (g), (h) and (i) example controllers are shown. In each case the nominal controller's dynamics are designed by tuning one of the parameters above. Other parameters are changed to compensate for any loss in expression or decoupling ability. See Table S2 for further details. (g) The impact on the circuit dynamics of increasing $\delta_{r}$. (h) The impact on the circuit dynamics of increasing $\delta_{p_{f}}$. (i) The impact on the circuit dynamics of increasing $\eta_{f}$. 


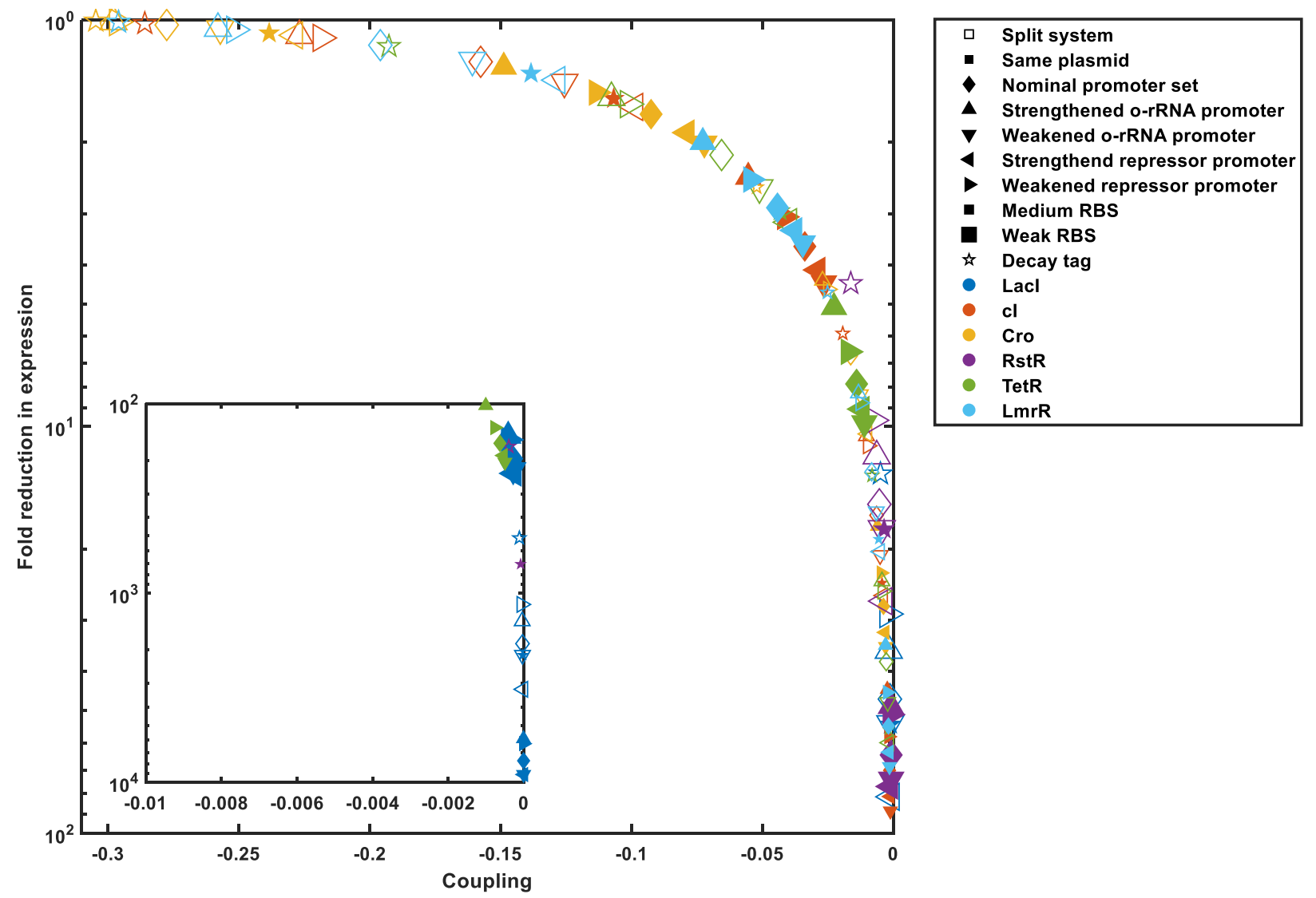

Figure 4: Comparison of biological implementations based on orthogonal repressors. Simulations of implementations based on the repressors in different plasmid confirmations and with degradation motifs. (a) The positions of the prototype controllers in the coupling-expression space. Inset, expansion of the main figure around point $(0,100)$. Point colours represent the regulator protein and point style denotes copy number as follows: Same plasmid, $g_{r, T}=g_{f, T}=100 \mathrm{nM}$; Chromosomal, $g_{r, T}=500 \mathrm{nM}$ and $g_{f, T}=10 \mathrm{nM}$. Decay tag, $g_{r, T}=500 \mathrm{nM}, g_{f, T}=10 \mathrm{nM}, \delta_{p_{f}}=3 \mathrm{~h}^{-1} \approx t_{1 / 2}=8$ minutes, equivalent to LVA tag. ${ }^{24}$ 

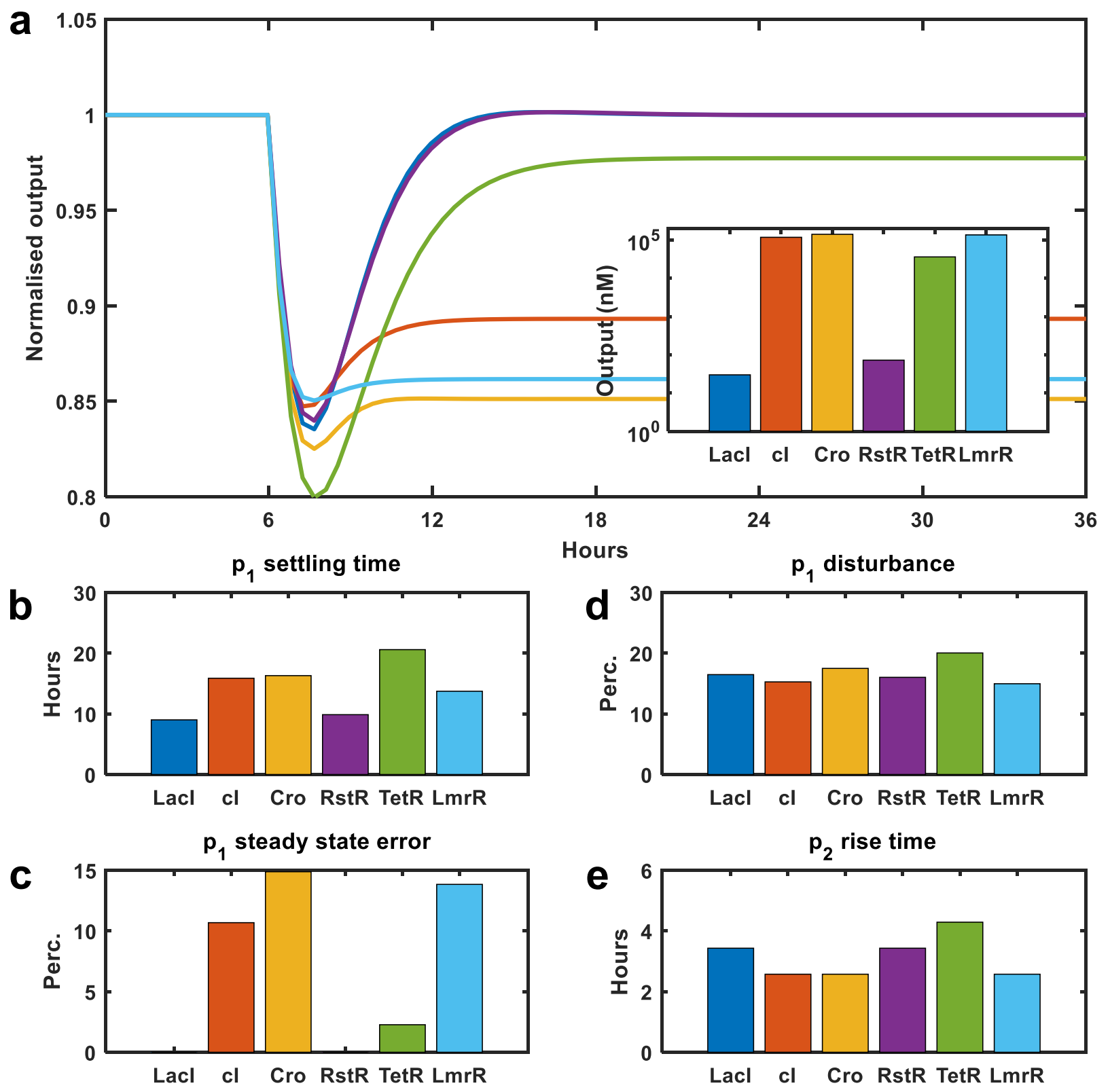

Figure 5: Dynamic responses of potential biological implementations. Characterisation of the response of $p_{1}$ to the disturbance caused by $u_{2}$. Designs are available in Table S5. (a) Circuit dynamics showing the normalised levels of protein 1. Inset, steady state output at $\mathrm{t}=48 \mathrm{~h}$. (b) $p_{1}$ settling time. The number of hours from the induction until $p_{1}$ returns to steady state. (c) $p_{1}$ steady state error. The difference between $p_{1}(t=48)$ and $p_{1}(t=12)$. (d) $p_{1}$ disturbance. The percentage fall in $p_{1}$ upon $u_{2}$ induction. (e) $p_{2}$ rise time. The time it takes for $p_{2}$ to increase from $10 \%$ of its steady state to $90 \%$ of its steady state. 

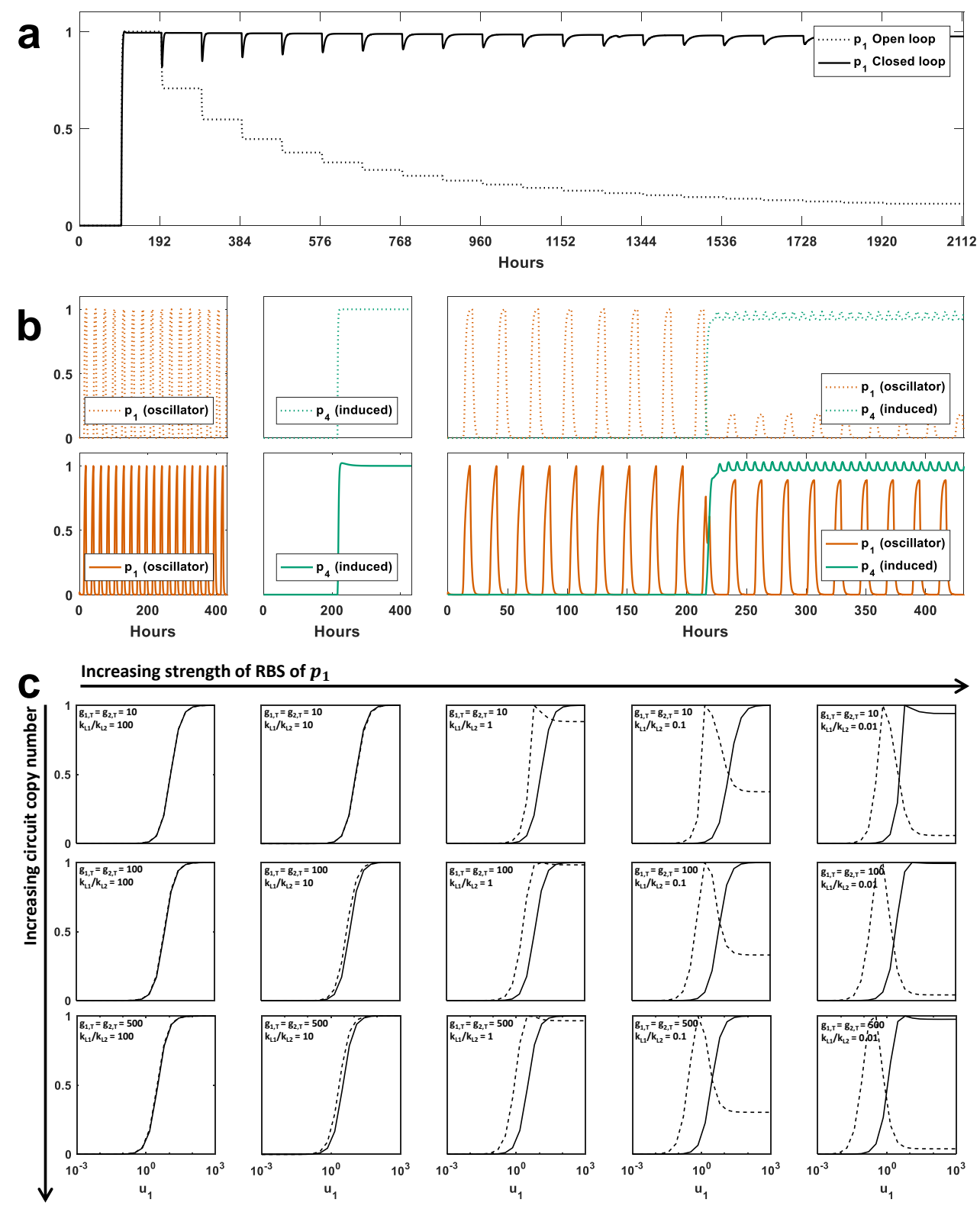

Figure 6: The controller rescues modularity in a variety of circuit contexts A range of common circuits were simulated in both the open and closed loop confirmations. All y-axes are normalised output. (a) The controller successfully renders a gene invulnerable to the induction of many additional genes at $100 \mathrm{~h}$ intervals. Other genes not shown.(b) Maintaining repressilator behaviour in the presents of an induced gene. The repressilator (protein $p_{1}$ to $p_{3}$, only $p_{1}$ is shown) is simulated before an additional gene $p_{4}$ with a stronger RBS is induced. Upper panels Open loop (no controller). Lower panels Closed loop (with controller). Left and centre panels Function of the individual modules alone. Right Function of the two modules in one circuit. $p_{4}$ is induced at $24 \mathrm{~h}$. (c) The controller removes resource limitation-induced failure in the design of an activation cascade $\left(u_{1} \rightarrow p_{1} \rightarrow p_{2}\right)$. In the absence of the controller (dashed line) some prototype designs do not show the monotonically increasing output of $p_{2}$ to $u_{1}$ as desired in an activation cascade. The controller removes these resource limitations allowing the circuit to function as expected across all prototype designs. 
Graphical TOC Entry

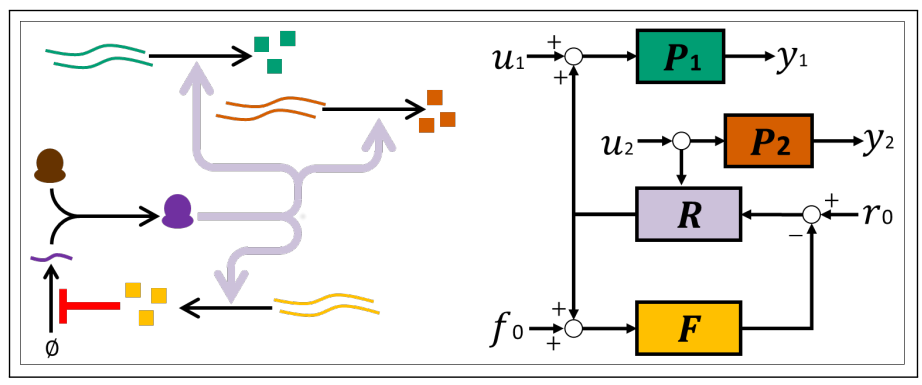

